\title{
Torakolomber omurga kırıklarında cerrahi ne zaman gerekli?
}

\section{When is surgery necessary for thoracolumbar spine fractures?}

\author{
H. Bahadır Gökçen ${ }^{1}$, Çağatay Öztürk² \\ ${ }^{1}$ İstinye Üniversitesi, Bahçelievler Medicalpark Hastanesi, İstanbul \\ ${ }^{2}$ Istinye Üniversitesi, LIV Hospital, İstanbul
}

Omurga kırıkları içinde torakolomber bölge kırıkları sık görülen zedelenmelerdir. Torakal ve lomber bölgenin geçiş zonunda yer aldıklarından, uygun tedavi edilmediklerinde ileride deformite geliştirme ve nörolojik komplikasyonlara yol açma ihtimali yüksektir. Konservatif ya da cerrahi tedavi seçenekleri vardır. Tedavi seçeneklerini belirlerken kırık tipini ve zedelenmenin morfolojisini anlamak adına kırık sınıflamasını yapmak önem teşkil eder. Cerrahi tedavi endikasyonlarını belirlerken dikkat edilmesi gereken önemli bazı kavramlar vardır. Bunlar stabilite kavramı ve nörolojik zedelenmenin eşlik edip etmemesidir. Stabilitede posterior ligamentöz kompleks önemli bir yer tutar. Posteriordaki bu kompleksin değerlendirilmesinde manyetik rezonans görüntüleme çok değerlidir. Kırığa bağlı norolojik komplikasyonlar ise birçok cerrah için cerrahi tedavi endikasyonu oluşturur. Cerrahide anterior yaklaşımlar, posterior yaklaşımlar veya kombine anterior-posterior yaklaşımlar uygulanabilir. Kırık tipi ve cerrahın tecrübesi cerrahi yaklaşımın seçilmesinde etkili faktörlerdir. Gelişen cerrahi implant teknolojisi ve cerrahi yaklaşımların modifikasyonları posteriordan yaklaşımlar ile spinal kanalın $360^{\circ}$ dekompresyonuna olanak sağlamıştır. Anterior yaklaşımın komplikasyon riskleri yüksek ve tecrübeye daha bağımlı bir cerrahi olması, son zamanlarda torakolomber omurga kırıklarında posterior yaklaşımları daha popüler hale getirmiştir. Bu makalede torakolomber omurga kırıklarının sınıflamasından ve cerrahi yaklaşımlarından bahsedilmiş olup cerrahinin hangi tip kırıklarda ve ne zaman gerektiği konuları tartışılmıştır.

Anahtar sözcükler: torakolomber; kırık; stabilite; cerrahi
Within the spine fractures, thoracolumbar region fractures are common injuries. Since they are located in the transition zone of the thoracic and lumbar region, if not properly treated they are more likely to cause deformity development and/ or neurological complications in the future. Conservative treatment and surgical treatment options are available. When determining the treatment option, it is important to classify the fracture in order to understand the type of fracture, and the morphology of the injury. There are some important concepts to be considered when determining the indications for surgical treatment. These are the concepts of stability and neurological impairment. Stabilized posterior ligamentous complex occupies an important place. Magnetic resonance imaging is very valuable for the evaluation of this complex in the posterior. Neurological complications related to fracture are an indication of surgical treatment for many surgeons. Anterior, posterior or combined anterior-posterior approaches may be applied surgically. The fracture type and experience of the surgeon are effective factors in selecting the surgical approach. Improved surgical implant technology and modifications of surgical approaches have allowed $360^{\circ}$ decompression of the spinal canal by posterior approach. The high complication risks of anterior surgery and the fact that it is a more experience dependent surgical technique has recently made posterior approach more popular in thoracolumbar spine fractures. In the current article, we mentioned the surgical approaches and the classification of thoracolumbar spine fractures, and discussed which fracture types require surgery.

Key words: thoracolumbar; fracture; stability; surgery
T orakolomber bölgenin kırıkları, en sık karşılaşılan omurga kırıkları arasındadır. Akut dönemde nörolojik komplikasyonlara yol açabileceği gibi, tedavi edilmemiş olgularda ilerleyen dönemde kifotik deformitelere de yol açabilir. Torakolomber bölgenin lomber ve torakal bölgenin geçiş zonunda yer alması, gelişebilecek komplikasyonları önlemek adına uygun tedavi edilmesini daha önemli hale getirir. Konservatif yöntemlerden, farklı cerrahi prosedürlere kadar değişen bir yelpazede tedavi algoritması vardır. Bu bölümde, bu bölgenin kırıklarında cerrahi tedavi gereksinimi ve cerrahi tedavinin zamanlaması tartışılacaktır.

Torakolomber omurga kırığı saptanan hastalarda, yaklaşık \%25 oranında farklı omurga segmentlerinde

- Iletişim adresi: Dr. H. Bahadır Gökçen, Edirne Çirpici Sokak No: 9, Topkapı, 34010 Zeytinburnu, İstanbul Tel: 0212 - 48416 40/+9, 0533 - 5742104 e-posta: bahadrgokcen@gmail.com

- Geliș tarihi: 1 Kasım 2018 Kabul tarihi: 1 Kasım 2018 
kırık olma ihtimali vardır. ${ }^{[1]}$ Bu bölgenin travmalarında nörolojik zedelenme riski yaklaşık \%15 oranındadır. Özellikle fleksiyon-distraksiyon zedelenmelerinde eşlik eden iç organ yaralanmaları görülme olasılığı da vardır ve bu oran nerdeyse yarı yarıyadır. ${ }^{[2]} \mathrm{Bu}$ nedenle, yaralanma mekanizmasını anlamak ve sınıflamasını yapmak klinik açıdan önem teşkil eder.

\section{TORAKOLOMBER OMURGA KIRIKLARINDA SINIFLAMA}

ilk olarak Watson-Jones 1938 yılında bir sınıflama yapmıştır. ${ }^{[3]}$ Daha sonra Holdsworth iki kolon teorisi kullanarak ${ }^{[4]}$, Denis ise üç kolon teorisi kullanarak birer sınıflama yapmışlardır. ${ }^{[5]}$ Denis'in sınıflamasında, orta kolonun stabilitedeki önemine yer verilmiş ve orta kolon zedelenmeleri instabil kırıklar olarak değerlendirilmiştir. AO (Association for Osteosynthesis) sınıflamasında ise kompresyon zedelenmeleri Tip A kırıklar olarak adlandırılır; Tip B kırıklar distraksiyon zedelenmeleri, Tip C ise kırıklı-çıkık olarak sınıflandırılmıştır.

AO sınıflamasında zedelenme mekanizması, kırık morfolojisi ve mekanik stabilite esas alınmıştır. Sınıflamanın tedaviyi yönlendirmedeki eksikliği, nörolojik durum hakkında bilgi vermemesidir. Vaccaro ve ark., nörolojik durumu da içeren bir sınıflama sistemi geliştirmiştir. Bu sınıflama sisteminin diğer sınıflama sistemlerinden farkı ise tedavi için de bir yönlendirme yapmasıdır. Bu sınıflama sistemine göre yapılan puanlamada, beş puan üzerinde alınan puanlar tedavinin cerrahi yapılmasının daha uygun olabileceğini gösterir.

\section{STABILITE KAVRAMI VE NÖROLOJIK DEFISITIN ÖNEMI}

Torakolomber omurga zedelenmelerinde genel kanıya göre, eğer hastanın muayenesinde nörolojik defisit var ve instabil bir kırık ise tedavi cerrahidir. Aslında bu, üzerinde çok tartışılacak bir konu değildir, fakat nörolojik olarak semptomu olmayan ve stabil torakolomber omurga kırıklarında tedavi yaklaşımı tartışmaya açıktır.

Torakolomber kırıklarda stabiliteyi değerlendirmek için, posterior ligamentöz kompleks (PLK)'in sağlamlığı hakkında bilgi edinmek gerekir. PLK; supraspinöz bağ, interspinöz bağ, ligamentum flavum ve faset eklemlerden oluşur. Sağlamlığını değerlendirmek için çekilen görüntüleme yöntemlerinde bazı bulgulara dikkat etmek gerekir. Direkt grafide vertebra cisim yüksekliğinde \%50'den fazla azalma olması, interspinöz aralıkta artış ve $30-35^{\circ}$ 'den fazla kifoz artışı PLK zedelenmesini düşündürür. ${ }^{[6-8]}$ Bilgisayarlı tomografide ise faset eklemleri değerlendirmek önemlidir. Faset eklemlerdeki açılma ve düzensizlik görünümü PLK zedelenmesi açısından değerlidir..$^{[9,10]}$ Fakat esas değerli olan görüntüleme yöntemi ise manyetik rezonans (MR) görüntülemedir. Özellikle T2 yağ baskılı kesitlerde PLK'de sinyal artışı görülmesi zedelenme lehine yorumlanır. MR değerlendirmenin PLK zedelenmesini değerlendirmede yüksek duyarlılı ve özgüllüğe sahip olduğu yayınlarda bildirilmiştir. ${ }^{[11-13]}$ Bu nedenle, torakolomber omurga kırıklarında tedavi kararı verilirken MR ile değerlendirmek önemlidir. Kırığa bağlı spinal kanal zedelenmesinin neden olduğu komplet veya inkomplet nörolojik defisiti olan hastalar, kırık tipinden veya PLK zedelenmesinden bağımsız olarak instabil kabul edilirler. ${ }^{[14]}$ Nörolojik zedelenmenin eşlik ettiği kırıklar bazı görüşlere göre kesin bir cerrahi endikasyon olmasa $\mathrm{da}^{[15-18]}$, nörolojik zedelenmenin ilerlememesi ve durumun düzeltilmesi amaçlanarak cerrahi uygulamak, klinik olarak kabul gören bir yaklaşımdır. Biz de kliniğimizde nörolojik zedelenmesi olan torakolomber omurga kırıklarında cerrahi tedavi seçeneğini ön planda tutmaktayız.

\section{TORAKOLOMBER OMURGA KIRIKLARINDA CERRAHI TEDAVI SEÇENEKLERi}

Anterior yaklaşımlar, posterior yaklaşımlar ve kombine anterior-posterior yaklaşımlar olarak üç ana başlık altında toplanabilir. Her ne kadar biz kendi pratiğimizde sıklıkla posterior yaklaşımları kullansak $\mathrm{da}$, anterior yaklaşımların da gerekli olduğu endikasyonlar vardır. Gelişen cerrahi implant teknolojisi ve cerrahi yaklaşım prosedürleri ile, anterior yaklaşımlar neredeyse yok denecek kadar azalmıştır. Fakat özellikle disk veya kırık parçasına bağlı spinal kanal basısı olan burst kırıklarında, posterior yaklaşım ile yeterli dekompresyon sağlanamayacağı düşünülüyor ise anterior yaklaşım gerekebilir. Anterior yaklaşım için bu cerrahiye alışık olmak ve tecrübeli olmak gerekmektedir. Literatürde, anterior yaklaşımın posterior yaklaşıma göre deformiteyi düzeltmede daha başarılı olduğunu bildiren yayınlar vardır. Anterior yaklaşımın posterior yaklaşıma göre daha iyi bir stabilite sağladığından da bahsedilmiştir. ${ }^{[19-22]}$ Özellikle lomber yerleşimli omurga kanalını baskılayan burst kırıklarında anterior-posterior kombine cerrahi, tercih edilmesi gereken yaklaşımdır (Şekil 1). Fakat, anterior yaklaşımın yukarıda bahsettiğimiz gibi avantajları olsa da, iç organ yaralanması ve damar yaralanması gibi daha kötü sonuçlar doğurabilecek ciddi komplikasyonlara neden olabilir. ${ }^{[14]}$ Posterior yaklaşım geleneksel bilgi olarak daha çok nörolojik defisiti olmayan torakolomber omurga kırıklarında kullanılsa da, bahsettiğimiz üzere, gelişen implant teknolojisi ve cerrahi yaklaşımlardaki modifikasyonlar bu yaklaşımın spinal kanalın 

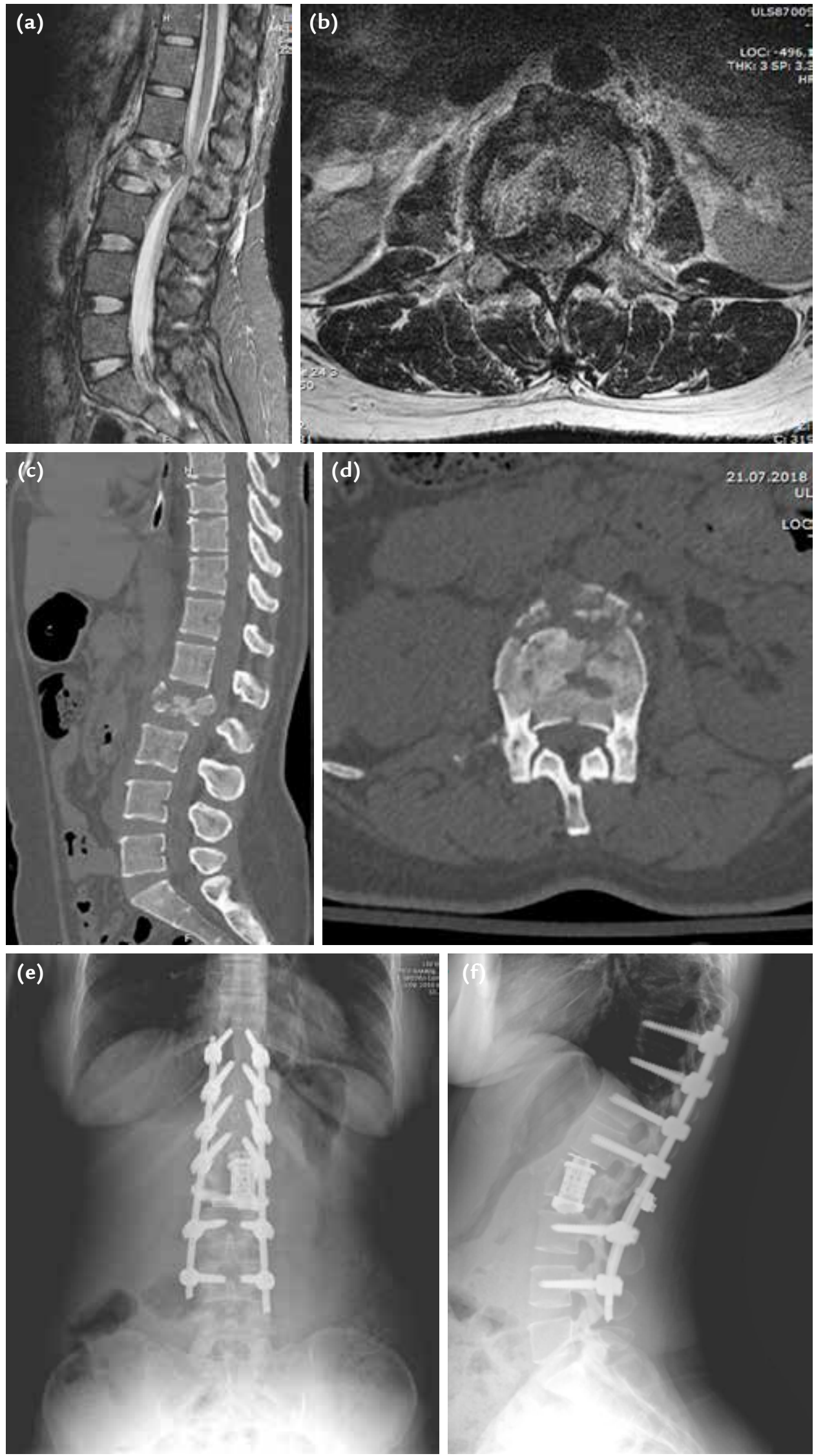

Şekil 1. a-f. Otuz beş yaşında bayan hasta, yüksekten düşme (suisid) sonrası merkezimize sevk edildi. Sagittal (a) ve aksiyel (b) MR kesitlerinde L2 burst kırı̆gı ve ciddi spinal kanal işgali mevcut. Hastanın nörolojik tablosu normal. Sagittal (c) ve aksiyel (d) BT kesitlerinde burst kırı̆ııın şekli daha net seçilebilmekte. Hastaya posterior enstrümantasyon, posterior laminektomi ile dekompresyon, anterior L2 korpektomi, dekompresyon, kafes ile füzyon ameliyatı uygulandı $(e, f)$. 
$360^{\circ}$ dekompresyonu için de kullanılabilecek bir yöntem olmasını sağlamıştır. Dekompresyon, indirekt mekanizma olan "ligamentotaksis" veya direkt mekanizma olan posteriordan laminektomi yöntemi ile sağlanabilir. Posterior yaklaşımda $360^{\circ}$ dekompresyona olanak veren prosedür, posterior vertebral kolon rezeksiyonudur. Bu prosedür ile kırık vertebra segmentinin çıkartılması, spinal kanalın hem önden hem de arkadan dekompresyonunu sağlar. Özellikle instabil burst (patlama) kırıklarında, kırıklı çıkık torakolomber omurga yaralanmalarında ve anterior cerrahi gerektiren fakat anterior cerrahinin hastaya vereceği medikal yükten kaçınıldığı durumlarda uygun bir yaklaşımdır. Fakat, en az anterior cerrahi kadar tecrübe gerektirir.

Posterior yaklaşımlarda tartışılan bir konu; füzyonun kısa segment mi, yoksa uzun segment mi yapılması gerektiğidir. Kısa segment füzyon özellikle fleksiyon-distraksiyon zedelenmelerinde önerilen bir tedavi yaklaşımıdır. ${ }^{[14]}$ Perkütan pedikül vidası uygulaması ile kısa segment füzyonsuz yaklaşımlar bu kırıklarda kullanılabilir. Literatürde nörolojik defisit eşlik etmeden fleksiyon-distraksiyon zedelenmesi olan hastalarda uygulanan bu yöntem ile iyi sonuçlar alındığı bildirilmiştir. ${ }^{[23]}$ Başka bir çalışmada da, perkütan pedikül vidası uygulamanın açık yöntem kadar etkili olduğundan bahsedilmiştir. ${ }^{[24]}$ Kısa segment füzyonun uzun segmente göre daha fazla redüksiyon kaybına ve implant yetersizliğine yol açtığına dair yayınlar da bildirilmiştir. ${ }^{[25-27]}$ Aslında burada önemli olan, kısa segment füzyonun hangi kırıklar için daha uygun olduğudur. AO sınıflamasına göre, A3 ve B2 kırıklarda kifoplasti ile desteklendiğinde iyi cerrahi sonuçların alındığı bildirilmiştir. Ayrıca, kırık vertebra segmentine de pedikül vidası koymanın stabiliteyi arttırdığı bildirilmiştir. ${ }^{[28-30]}$

\section{SONUÇ}

Torakolomber omurga kırıkları, omurga zedelenmeleri içinde sık rastlanılan kırıklar olup, kırık tipini anlamak ve eşlik eden nörolojik zedelenme riskini belirlemek tedavi algoritması oluşturmakta önemlidir. Kırığın stabilitesini anlamak için, PLK yaralanması olup olmadığını değerlendirmek tedavi yaklaşımı açısından çok önemli bir yere sahiptir. Bu nedenle, torakolomber kırıklarda PLK incelenmesi için MR görüntüleme önemli bir yere sahiptir. İnstabilite kavramında ayrıca; faset eklemlerin değerlendirilmesi, \%50'den fazla kanal içi işgalinin olması, kırık bölgesinde $30^{\circ}$ 'den fazla lokal kifozun ölçülmesi ve vertebra cisminde \%50'den fazla çökmenin görülmesi de önem arz etmektedir. Günümüzde bu kırıkların cerrahi tedavisi posterior yollar ile yapılabilmekle birlikte, anterior cerrahinin de gerekli olabileceği akılda tutulmalıdır. Anterior cerrahi gereken durumlarda; bu yaklaşımda tecrübeli olmak görülebilecek ciddi komplikasyonların oranını azaltabilir. Cerrahın, torakolomber omurga kırıklarının tedavisinde; uygulamayı planladığı cerrahinin avantajları ve dezavantajlarını iyi değerlendirdiğinden ve tecrübesinin de el verdiği ölçüde gerekli yaklaşımı uygulayabileceğinden emin olması gerekir.

\section{KAYNAKLAR}

1. Calenoff L, Chessare JW, Roger LF, Toerge J, Rosen JS. Multiple level spinal injuries: importance of early recognition. AJR Am J Roentgenol 1978;130(4):665-9. Crossref

2. Eismont F. Flexion-distraction Injuries of the thoracic and lumbar spine. In: Levine AM, Eismont FJ, Garfin SR, Zigler JE, editors. Spine Trauma. Philadelphia, PA: WB Saunders; 1998.

3. Watson-Jones R. The results of postural reduction of fractures of the spine. J Bone Joint Surg Am 1938;20:567-86.

4. Holdsworth F. Fractures, dislocations and fracturedislocations of the spine. J Bone Joint Surg Am 1970;52(8):1534-51. Crossref

5. Denis $F$. The three-column spine and its significance in the classification of acute thoracolumbar spinal injuries. Spine 1983;8(8):817-31. Crossref

6. Benson DR, Burkus JK, Montesano PX, Sutherland TB, McLain RF. Unstable thoracolumbar and lumbar burst fractures treated with the AO fixateur interne. J Spinal Disord 1992;5(3):335-43. Crossref

7. McAfee PC, Yuan HA, Fredrickson BE, Lubicky JP. The value of computed tomography in thoracolumbar fractures. An analysis of one hundred consecutive cases and a new classification. J Bone Joint Surg Am 1983;65(4):461-73. Crossref

8. Reid DC, Hu R, Davis LA, Saboe LA. The nonoperative treatment of burst fractures of the thoracolumbar junction. J Trauma 1988;28(8):1188-94. Crossref

9. Schweitzer KM, Vaccaro AR, Harrop JS, Hurlbert J, Carrino JA, Rechtine GR, Schwartz DG, Alanay A, Sharma DK, Anderson DG, Lee JY, Arnold PM. Interrater reliability of identifying indicators of posterior ligamentous complex disruption when plain films are indeterminate in thoracolumbar injuries. J Orthop Sci 2007;12(5):437-42. Crossref

10. Lee JY, Vaccaro AR, Schweitzer KM Jr, Lim MR, Baron EM, Rampersaud R, Öner FC, Hulbert RJ, Hedlund R, Fehlings MG, Arnold P, Harrop J, Bono CM, Anderson PA, Patel A, Anderson DG, Harris MB. Assessment of injury to the thoracolumbar posterior ligamentous complex in the setting of normal-appearing plain radiography. Spine J 2007;7(4):422-7. Crossref

11. Petersilge CA, Pathria MN, Emery SE, Masaryk TJ. Thoracolumbar burst fractures: evaluation with MR imaging. Radiology 1995;194(1):49-54. Crossref

12. Lee HM, Kim HS, Kim DJ, Suk KS, ParkJO, Kim NH. Reliability of magnetic resonance imaging in detecting posterior ligament complex injury in thoracolumbar spinal fractures. Spine (Phila Pa 1976) 2000;25(16):2079-84. Crossref

13. Haba H, Taneichi $H$, Kotani $Y$, Terae S, Abe S, Yoshikawa $H$, Abumi K, Minami A, Kaneda K. Diagnostic accuracy of magnetic resonance imaging for detecting posterior ligamentous complex injury associated with thoracic and lumbar fractures. J Neurosurg 2003;99(1):20-6. Crossref

14. Kim BG, Dan JM, Shin DE. Treatment of thoracolumbar fracture. Asian Spine J 2015;9(1):133-46. Crossref 
15. Andreychik DA, Alander DH, Senica KM, Stauffer ES. Burst fractures of the second through fifth lumbar vertebrae. Clinical and radiographic results. J Bone Joint Surg Am 1996;78(8):1156-66. Crossref

16. Dai LY, Jiang LS, Jiang SD. Conservative treatment of thoracolumbar burst fractures: a long-term follow-up results with special reference to the load sharing classification. Spine (Phila Pa 1976) 2008;33(23):2536-44. Crossref

17. Knight RQ, Stornelli DP, Chan DP, Devanny JR, Jackson KV. Comparison of operative versus nonoperative treatment of lumbar burst fractures. Clin Orthop Relat Res 1993;(293):112-21. Crossref

18. Moller A, Hasserius R, Redlund-Johnell I, Ohlin A, Karlsson MK. Nonoperatively treated burst fractures of the thoracic and lumbar spine in adults: a 23- to 41-year follow-up. Spine J 2007;7(6):701-7. Crossref

19. Hitchon PW, Torner J, Eichholz KM, Beeler SN. Comparison of anterolateral and posterior approaches in the management of thoracolumbar burst fractures. J Neurosurg Spine 2006;5(2):117-25. Crossref

20. Sasso RC, Renkens K, Hanson D, Reilly T, McGuire RA Jr, Best NM. Unstable thoracolumbar burst fractures: anterior-only versus short-segment posterior fixation. J Spinal Disord Tech 2006;19(4):242-8. Crossref

21. Shono Y, McAfee PC, Cunningham BW. Experimental study of thoracolumbar burst fractures. A radiographic and biomechanical analysis of anterior and posterior instrumentation systems. Spine (Phila Pa 1976) 1994;19(15):1711-22. Crossref

22. Gurwitz GS, Dawson JM, McNamara MJ, Federspiel CF, Spengler DM. Biomechanical analysis of three surgical approaches for lumbar burst fractures using short-segment instrumentation. Spine (Phila Pa 1976) 1993;18(8):977-82. Crossref
23. Kim YM, Kim DS, Choi ES, Shon HC, Park KJ, Cho BK, Jeong JJ, Cha YC, Park JK. Nonfusion method in thoracolumbar and lumbar spinal fractures. Spine (Phila $\mathrm{Pa}$ 1976) 2011;36(2):170-6. Crossref

24. Grossbach AJ, Dahdaleh NS, Abel TJ, Woods GD, Dlouhy BJ, Hitchon PW. Flexion-distraction injuries of the thoracolumbar spine: open fusion versus percutaneous pedicle screw fixation. Neurosurg Focus 2013;35(2):E2. Crossref

25. Ebelke DK, Asher MA, Neff JR, Kraker DP. Survivorship analysis of VSP spine instrumentation in the treatment of thoracolumbar and lumbar burst fractures. Spine (Phila Pa 1976) 1991;16(Suppl):S433. Crossref

26. McLain RF, Sparling E, Benson DR. Early failure of shortsegment pedicle instrumentation for thoracolumbar fractures. A preliminary report. J Bone Joint Surg Am 1993;75(2):1627. Crossref

27. McNamara MJ, Stephens GC, Spengler DM. Transpedicular short-segment fusions for treatment of lumbar burst fractures. J Spinal Disord 1992;5(2):183-7. Crossref

28. Chen C, Lv G, Xu B, Zhang X, Ma X. Posterior short-segment instrumentation and limited segmental decompression supplemented with vertebroplasty with calcium sulphate and intermediate screws for thoracolumbar burst fractures. Eur Spine J 2014;23(7):1548-57. Crossref

29. Guven O, Kocaoglu B, Bezer M, Aydin N, Nalbantoglu $U$. The use of screw at the fracture level in the treatment of thoracolumbar burst fractures. J Spinal Disord Tech 2009;22(6):417-21. Crossref

30. Mahar A, Kim C, Wedemeyer M, Mitsunaga L, Odell T, Johnson B, Garfin S. Short-segment fixation of lumbar burst fractures using pedicle fixation at the level of the fracture. Spine (Phila Pa 1976) 2007;32(14):1503-7. Crossref 